\title{
A SEARCH FOR [NE II] 12.8 MICRON LINE EMISSION FROM GALACTIC ULTRACOMPACT H II REGIONS
}

\author{
H. MATSUHARA, H. TAKAHASHI AND H. WATARAI \\ Department of Astrophysics, Nagoya University \\ Furo-cho, Chikusa-ku, Nagoya 464-01, Japan
}

\section{Introduction}

Starburst galaxies are powered by newly formed massive stars, and are heavily obscured by dust, prohibiting the UV and optical studies. A finestructure line from $\mathrm{Ne}^{+}$ion ([Ne II] $12.8 \mu \mathrm{m}$ ) is usually the brightest midinfrared(MIR) line emitted from starburst galaxies. The [Ne II] line suffers from far less dust extinction than the optical line does, and hence it is often used as a probe for starburst properties of galaxies(Achtermann \& Lacy, 1995; Roche et al., 1991). However, the [Ne II] line should not be used as the physical probe unless the relationship between $\mathrm{Ne}^{+}$abundance and spectral type of the ionizing star is established for very dusty $H$ II regions. To study this, we started the [Ne II] line survey toward dusty ultracompact(UC) $\mathrm{H}$ II regions in our Galaxy. The UC H II regions are powered by newly formed early-type stars whose spectral type can be inferred from their radio and far-infrared(FIR) properties.

\section{Observations and Results}

[Ne II] $12.8 \mu$ m line observations have been made with the $2.3 \mathrm{~m}$ telescope at Wyoming Infrared Observatory (WIRO) on 15-18 March 1997, using a MIR Fabry-Perot Imager(MIRFI) developed at Nagoya University(Watarai et al., 1996). MIRFI provides the [Ne II] line flux with spectral resolving power of 1500 over a field of 10 " $\times 10$ " with a plate scale of 2 " $/ \operatorname{pixel}(5 \times 5$ pixel).

Among the 9 UC H II regions observed so far, the [Ne II] emission was detected from 6 regions. Then the [Ne II] flux is corrected for the interstellar extinction, and using the $2 \mathrm{~cm}$ continuum flux obtained from 
radio VLA observations(Wood \& Churchwell, 1989; Kurtz et al., 1994), the $\mathrm{Ne}^{+}$abundance $\left(\left[\mathrm{Ne}^{+} / \mathrm{H}^{+}\right]\right)$is derived. The results are shown in table 1 , together with spectral type of ionizing star inferred from FIR and radio properties(mostly from Wood \& Churchwell and Kurtz et al.). The values with an asterisk are preliminary ones.

TABLE 1. Observed UC H II regions and their $\mathrm{Ne}^{+}$abundance

\begin{tabular}{lcccc}
\hline Name & $\begin{array}{c}\text { Galactocentric } \\
\text { Distance }[\mathrm{kpc}]\end{array}$ & $\begin{array}{c}\text { Interstellar } \\
\text { Extinction(Av)[mag] }\end{array}$ & $\begin{array}{c}\text { Spectral Type } \\
\text { FIR / radio }\end{array}$ & $\begin{array}{c}{\left[\mathrm{Ne}^{+} / \mathrm{H}^{+}\right]} \\
\times 10^{4}\end{array}$ \\
\hline G29.96-0.02 & 4.4 & 15 & O5 / O5.5 & 0.78 \\
W51d & 6.7 & 20 & O4.5 / & 0.10 \\
K3-50A & 9.4 & 30 & O6 / O5.5 & $0.03^{*}$ \\
Mon R2 & 9.3 & 23 & $-/ \mathrm{B} 0$ & $0.03^{*}$ \\
G35.20-1.74 & 6.2 & 23 & O9.5 / O9 & $0.22^{*}$ \\
G45.12+0.13 & 7.1 & 25 & O5.5 / O6.5 & $0.26^{*}$ \\
G133.95+1.06 & 11. & 6 & B0 / O9.5 & $<0.01$ \\
G192.58-0.04 & 11. & 5 & B1 / B0.5 & $<2.0$ \\
G76.38-0.62 & 8.3 & 22 & B2 / B1 & $<1.4$ \\
\hline
\end{tabular}

\section{Discussion}

The [Ne II] emission was not detected from UC H II regions powered by the $\mathrm{B} 0$ and later $\mathrm{B}$ stars. This is reasonable because the ionization potential of $\mathrm{Ne}^{0}(21.56 \mathrm{eV})$ is higher than that of $\mathrm{H}(13.6 \mathrm{eV}$ ), and thus $\mathrm{B} 0$ (and later $\mathrm{B}$ ) stars cannot produce $\mathrm{Ne}^{+}$regions. On the other hand, if the spectral type is 07 or earlier than 07 , one would expect that $\mathrm{Ne}^{++}$is a major species and hence the $\mathrm{Ne}^{+}$abundance is relatively small. However, the results does not show such a trend. In addition, 2"/pixel spatial resolving power of MIRFI now enables us to investigate spatial distribution of $\left[\mathrm{Ne}^{+} / \mathrm{H}^{+}\right]$for some objects. This will be described in Watarai et al., 1997.

\section{References}

Achtermann, J. M., \& Lacy, J. H. (1995) Astrophys. J., 439, p.163

Kurtz, S., Churchwell, E., \& Wood, D. O. S. (1994), Astrophys. J. Suppl., 91, p.659

Roche, P. F., Aitken, D. K., Smith, C. H., \& Ward, M. J. (1991) Mon. Not. Roy. Astron. Soc., 248, p.606

Watarai, H., Hayata, E., Matsumoto, T., Takahashi, H., Tutui, Y., Yoda, H., Matsuhara, H., \& Greene, T. P. (1996), Pub. Astron. Soc. Pacific, 108, p.1033

Watarai, H., Matsuhara, H., Takahashi, H., \& Matsumoto, T. (1997), in preparation

Wood, D. O. S., \& Churchwell, E. (1989), Astrophys. J. Suppl., 69, p.831 